\title{
Análisis termodinámico de las condiciones de operación de los ciclos combinados turbina de gas-vapor con uno, dos y tres niveles de presión
}

\section{Thermodynamics analysis of the operation conditions of gas-steam turbine combined cycles with one, two and three pressure levels}

\author{
MÉNDEZ-CRUZ, Ladislao Eduardo ${ }^{1 * \dagger}$, SALAZAR-PEREYRA, Martín ${ }^{1}$, LUGO-LEYTE, Raúl ${ }^{2}$ y \\ PÉREZ-BEDOLLA, Rafael ${ }^{1}$ \\ ${ }^{1}$ Posgrado en Eficiencia Energética y Energías Renovables, Tecnológico de Estudios Superiores de Ecatepec, Av. \\ Tecnológico s/n, Valle de Anáhuac, Ecatepec de Morelos, Estado de México, México. \\ ${ }^{2}$ Departamento de Ingeniería de Procesos e Hidráulica, Universidad Autónoma Metropolitana - Iztapalapa, Avenida San \\ Rafael Atlixco 186, Col. Vicentina, 09340, Iztapalapa, CDMX, México.
}

ID $1^{\mathrm{er}}$ Autor: Ladislao Eduardo, Méndez-Cruz / ORC ID: 0000-0002-4329-0875, CVU CONACYT ID: 867424

ID $1^{\text {er }}$ Coautor: Martín, Salazar-Pereyra / ORC ID: 0000-0001-6487-3087, CVU CONACYT ID: 104009

ID $2^{\text {do }}$ Coautor: Raúl, Lugo-Leyte / ORC ID: 0000-0001-6578-0691, CVU CONACYT ID: 4941

ID $3{ }^{\text {er }}$ Coautor: Rafael, Pérez-Bedolla / ORC ID: 0000-0002-3715-3452, CVU CONACYT ID: 291170

DOI: $10.35429 / J M E .2019 .10 .3 .7 .18$

Recibido 25 Abril, 2019; Aceptado 25 Junio, 2019

\section{Resumen}

La tecnología de los ciclos combinados turbinas de gasvapor es una de las opciones más viables para la generación de energía eléctrica con menores costos de producción por kilowatt-hora y ambientales, considerando que el tipo de combustible sea gas natural. En la República Mexicana, las proyecciones de generación de energía al 2018 a través del uso de esta tecnología, establecen la posibilidad de instalar nuevas plantas de nueva generación con presiones y temperaturas de 100 a 160 bar y $538^{\circ} \mathrm{C}$ a $580^{\circ} \mathrm{C}$. Otra opción es la repotenciación de las centrales termoeléctricas y de los proyectos de cogeneración turbinas de gas-vapor. Por tal motivo, se realiza en este trabajo un análisis termodinámico de las configuraciones de los ciclos combinados de uno, dos y tres niveles de presión con el objetivo de establecer un análisis paramétrico de la capacidad de potencia generada, así como su eficiencia térmica y consumo térmico unitario con base a las características de la turbina de gas o vapor, así mismo se considera como restricción la calidad del vapor de la última etapa de expansión para delimitar las presiones de los domos de vapor de cada nivel.

Turbina de gas, Ciclo combinado, Análisis termodinámico

\begin{abstract}
The technology of gas-steam turbine combined cycles is once of the most viable options for the generation of electric power with lower costs of production per kilowatthour and environmental, considering that the type of fuel should be gas natural. In the Mexican Republic, the projections of power generation to 2018 through the use of this technology, it establishes the possibility to install new power generation plants with pressures of 100 to $160 \mathrm{bar}$, and temperatures of 538 to $580^{\circ} \mathrm{C}$. Another option it's the repowering of the thermoelectric plants and cogeneration gas-steam turbines projects. Therefore, in this paper present a thermodynamic analysis of the configurations of combined cycles of one, two and three pressure levels with the objective in order to establish a parametric analysis of the power generation capacity, as well the thermal efficiency and Heat Rate based on the characteristics of the gas and steam turbines, likewise the quality of the last expansion stage has considered as a restriction to delimit the pressure of the steam domes of each level.
\end{abstract}

Gas turbine, Combined cycle, Thermodynamic analysis

Citación: MÉNDEZ-CRUZ, Ladislao Eduardo, SALAZAR-PEREYRA, Martín, LUGO-LEYTE, Raúl y PÉREZBEDOLLA, Rafael. Análisis termodinámico de las condiciones de operación de los ciclos combinados turbina de gas-vapor con uno, dos y tres niveles de presión. Revista de Ingeniería Mecánica. 2019. 3-10: 7-18

\footnotetext{
* Correspondencia al Autor (Correo electrónico: ladislao.eduardo@gmail.com)

$\dagger$ Investigador contribuyendo como primer Autor.
} 


\section{Introducción}

La constante y creciente demanda de energía en los últimos años ha tenido una gran repercusión en el medio ambiente principalmente. Aunado a la disminución de los recursos fósiles y el incremento en el costo de los mismos, se ha apostado por encontrar sistemas de generación de energía en los cuales exista la mayor capacidad del aprovechamiento de estos recursos. Si bien existe una amplia gama para la generación de energía a partir de fuentes renovables (la cual en conjunto ha ido incrementando su participación en la matriz energética), no se tiene una completa confiabilidad para poder apostar del todo por este rubro. Por tal motivo, es de una gran relevancia el estudio de la generación de energía a partir de centrales de potencia tales como carboeléctricas, termoeléctrica, turbinas de gas y el acoplamiento de estos dos últimos a partir de ciclos combinados principalmente.

En este sentido, las centrales de ciclo combinado (turbina de gas-vapor) son confiables debido a múltiples factores, alta flexibilidad operacional, es decir, este tipo de centrales pueden operar a condiciones de plena carga o a cargas parciales, dependiendo de la demanda de energía que se requiera. Así mismo, tienen las mayores eficiencias térmicas que pueden ser mayores al $60 \%$ dependiendo del tipo de tecnología, lo que se traduce en un mayor aprovechamiento de los combustibles fósiles, principalmente el gas natural.

Además los ciclos combinados tienen la capacidad de generar una mayor potencia comparada con plantas convencionales y alternas esto se debe al aprovechamiento de los gases de combustión provenientes de la turbina de gas en una Caldera de Recuperación de Calor (CRC), en donde a partir de los arreglos de los intercambiadores de calor que la integran existe una transferencia de calor de la corriente de los gases hacia la corriente del agua, produciendo un flujo de vapor la cual, al expandirse en una turbina de vapor se genera una potencia adicional a la generada por la turbina de gas. Como consecuencia de ello, al generar una mayor cantidad de potencia con una misma fuente de calor, existirá una disminución de los gases contaminantes provocando un menor impacto al medio ambiente.
Se han realizado estudios de la eficiencia de una planta de ciclo combinado basándose en la optimización de los parámetros operativos de la CRC. A partir de una función objetivo termodinámica, se conduce a un aumento de la eficiencia térmica cercana al $60 \%$. Otra forma de maximizar la eficiencia del ciclo combinado es a través de la correcta distribución de los intercambiadores de calor de la CRC utilizando herramientas de simulación y el análisis de la diferencia de temperaturas de pinch point.

En la actualidad, las centrales de ciclo combinado que son empleadas tienen la configuración de 2 o 3 niveles de presión con recalentamiento de vapor, con temperaturas de entrada a la CRC que oscilan entre 700 y $920 \mathrm{~K}$. El objetivo de los principales fabricantes es seguir incrementando la eficiencia en periodos de tiempo muy cortos, dicho objetivo se persigue con el aumento de la temperatura de los gases a la entrada de la turbina de gas principalmente.

Así mismo también se centran en el estudio de la CRC, con la optimización de los parámetros operativos como flujo másico, perfil de temperaturas, los valores de presión de operación y la eficacia en los intercambiadores de calor. También se hace énfasis es en el uso de secciones de intercambiadores de calor con dos o más flujos paralelos de agua en donde exista un intercambio de calor con el flujo de los gases de combustión, únicamente cuando las corrientes de agua tengan las mismas propiedades térmicas.

Kaviri (2013) analizó el efecto que tiene la relación del flujo y temperatura de los gases a la salida de la turbina de gas sobre la eficiencia térmica del ciclo. Encontró que al incrementarse dicha temperatura, afecta de manera positiva la eficiencia del ciclo, teniendo como límite los $650^{\circ} \mathrm{C}$ ya que, sobrepasando esta temperatura existe una disminución de la eficiencia térmica. Ataei y Yoo (2010) investigó que, en las centrales de ciclo combinado existe un efecto de la diferencia de temperaturas de pinch point sobre la eficiencia térmica global, con la disminución de la diferencia de temperaturas de pinch point, existe una relación de la transferencia de calor mayor, lo que conlleva a una mayor eficiencia. Además, la transferencia de calor entre los gases de combustión y el agua puede ser incrementada con la instalación de más intercambiadores de calor. 
De acuerdo con la Prospectiva del Sector Eléctrico 2018-2032, la demanda de la energía mundial fue de 708 TWh para el año 2017. Del total de generación mundial, las energías renovables concentraron el $25 \%$, mientras que el $75 \%$ de la generación continúa siendo a partir de combustibles fósiles. En el caso de México, el consumo de energía tiene un crecimiento medio anual del $2.6 \%$ lo que represento una demanda de 293,127 GWh para ese mismo año. Así mismo, el Sector Eléctrico Nacional registro un total de 797 centrales de generación eléctrica de las cuales 526 corresponden a tecnologías convencionales y 271 de tecnologías limpias. Esto se traduce que, el $78.9 \%$ de la generación eléctrica proviene de tecnologías convencionales de los cuales la participación de ciclo combinado es del 50.2\%; por otro lado la participación de las tecnologías limpias en conjunto solamente representaron el $21.1 \%$.

Sin embargo, se tiene un pronóstico de incremento de la demanda de energía, la cual es de $3.2 \%$ anual entre el año 2018 y 2032; por esta razón se debe adicionar una capacidad de generación de energía de 66,912 MW de los cuales tenga un incremento la participación de energías limpias, representando el 54.9\% mientras que la parte restante sea a partir de tecnologías convencionales, teniendo casi en su totalidad al ciclo combinado con el $42.0 \%$. Una vez considerado la infraestructura actual junto con las adiciones en la capacidad, se prevé que para el año 2032, exista una capacidad de generación de energía de 130,292 MW, teniendo como líder en la participación de las energías convencionales a la tecnología del ciclo combinado.

Así mismo, debido a los grandes beneficios que conlleva la operación de los ciclos combinados, ha aumentado su implementación recientemente a lo largo de todo el mundo.

Una CRC puede tener hasta tres niveles de presión, los cuales se denominan domos baja, intermedia y alta presión. Cada nivel de presión tiene una configuración con tres principales intercambiadores de calor, economizador, evaporador y sobrecalentador. Integrar un mayor número de intercambiadores de calor, puede generar una mayor cantidad de vapor que finalmente se traduce en una mayor generación de potencia.
Por tal motivo, se realiza en este trabajo un análisis termodinámico de las configuraciones de los ciclos combinados de uno, dos y tres niveles de presión con el objetivo de establecer un análisis paramétrico de la capacidad de potencia generada, así como su eficiencia térmica y consumo térmico unitario con base a las características de la turbina de gas o vapor, con la finalidad de determinar la viabilidad y competitividad de la generación de energía a partir de un mayor aprovechamiento de los combustibles fósiles, en comparación con otros tipos de centrales convencionales. Las condiciones ambientales que se tomaron para este análisis fueron una presión atmosférica de 1.013 bar y una temperatura ambiente de $25^{\circ} \mathrm{C}$. En las Figuras 1 a 3 se muestran las tres configuraciones de ciclo combinado a analizar. Se muestra que a medida que se van incrementando los niveles de presión, existe una etapa adicional de la turbina de vapor. Además, se propone una misma configuración de la turbina de gas, con diferentes características tecnológicas, antigua (TG1), reciente (TG2) y de última tecnología (TG3), las cuales son acopladas a los ciclos combinados de uno, dos y tres niveles de presión respectivamente.

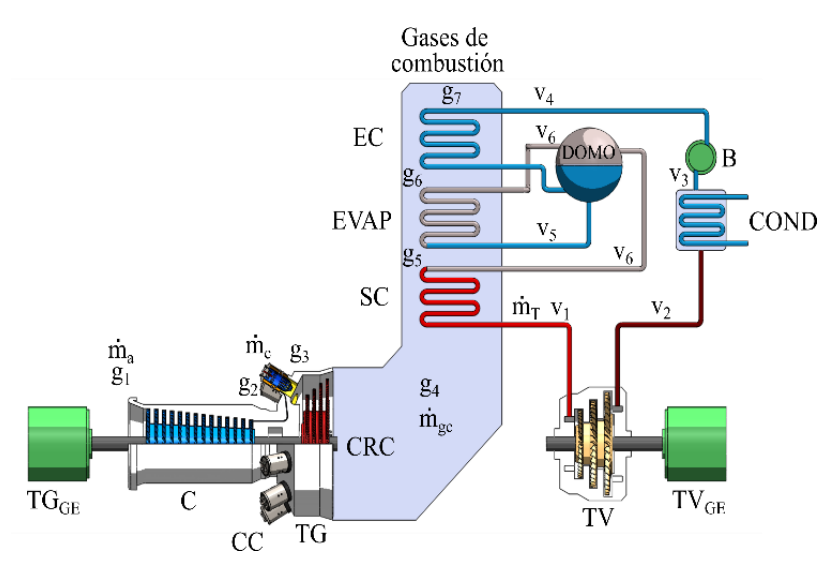

Figura 1 Ciclo Combinado de un nivel de presión

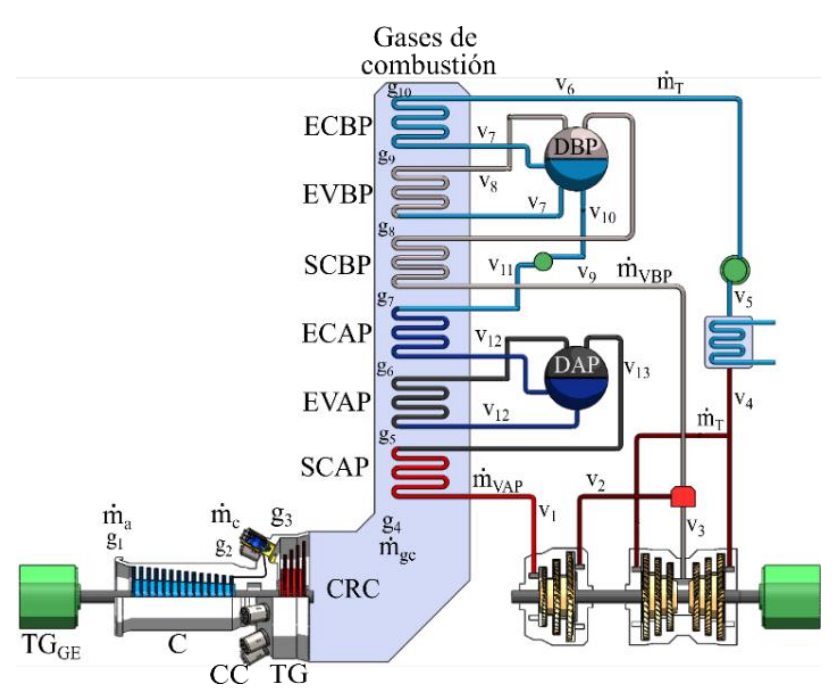

Figura 2 Ciclo Combinado de dos niveles de presión MÉNDEZ-CRUZ, Ladislao Eduardo, SALAZAR-PEREYRA, Martín, LUGO-LEYTE, Raúl y PÉREZ-BEDOLLA, Rafael. Análisis termodinámico de las condiciones de operación de los ciclos combinados termodinamico de las condiciones de operación de los ciclos combinados
turbina de gas-vapor con uno, dos y tres niveles de presión. Revista de turbina de gas-vapor con uno, dos y tres niveles de presión. Revista de
Ingeniería Mecánica. 2019. 
Se muestra que en las configuraciones de los ciclos combinados con uno y dos niveles de presión se tienen los intercambiadores de calor principales, Economizador, Evaporador y Sobrecalentador para las diferentes presiones.

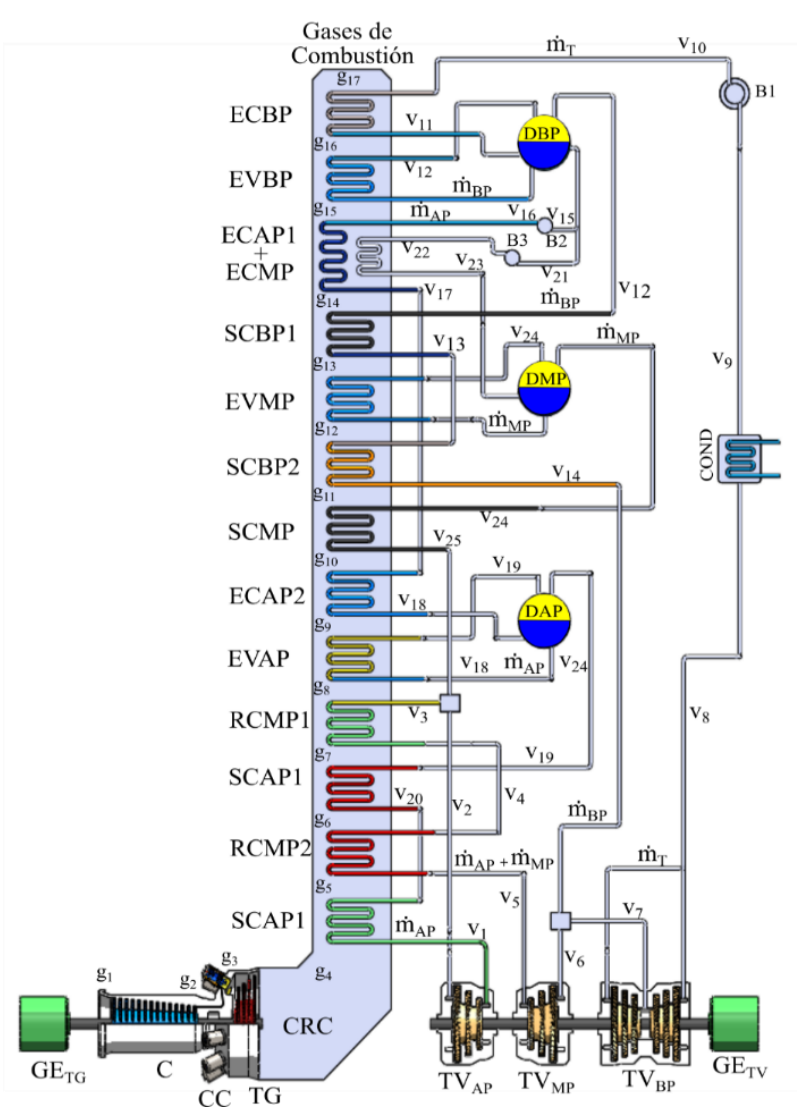

Figura 3 Ciclo Combinado de tres niveles de presión

Para el caso del ciclo combinado con tres niveles de presión, se puede mostrar que además de tener los mismos intercambiadores de calor con respecto a uno y dos niveles de presión, también existe un Recalentamiento a la presión de la turbina de media presión.

\section{Condiciones de operación del ciclo combinado}

\section{Turbina de gas}

En la Tabla 1 se muestran las condiciones de operación de tres diferentes configuraciones de turbinas de gas, todas ellas en función de parámetros tales como, relación de presiones, temperatura de entrada a la turbina de gas, potencia generada, eficiencia isoentrópica del compresor y de la turbina de gas.

Para este estudio, se considera una demanda de potencia constante de $150 \mathrm{MW}$, la cual debe ser generada por cada una de las configuraciones propuestas.

\section{TG1 TG2 TG3}

\begin{tabular}{|l|r|r|r|}
\hline$\pi,[-]$ & 10 & 14 & 16 \\
\hline $\mathrm{T}_{\mathrm{g} 3},\left[{ }^{\circ} \mathrm{C}\right]$ & 1100 & 1200 & 1400 \\
\hline$\dot{\mathrm{W}}_{\mathrm{mTG}},[\mathrm{MW}]$ & 50 & 75 & 150 \\
\hline$\eta_{\mathrm{SIC}},[-]$ & \multicolumn{4}{|r|}{0.88} \\
\hline$\eta_{\mathrm{SIT}},[-]$ & \multicolumn{3}{|r|}{0.9} \\
\hline$\dot{\mathrm{W}}_{\mathrm{TG}},[\mathrm{MW}]$ & \multicolumn{3}{|r|}{150} \\
\hline$\# \mathrm{TG},[-]$ & 3 & 2 & 1 \\
\hline $\mathrm{NP}_{\mathrm{CC}}[-]$ & 1 & 2 & 3 \\
\hline
\end{tabular}

Tabla 1 Condiciones de operación de la turbina de gas.

Debido a que en el análisis en la turbina de gas se realiza a partir de las propiedades del aire y de los gases de combustión, en la Tabla 2 se muestran las principales propiedades de ambas sustancias. Se considera una composición de un Gas Natural de $80 \% \mathrm{CH}_{4}, 15 \% \mathrm{C}_{2} \mathrm{H}_{6}$, y $4 \%$ $\mathrm{C}_{3} \mathrm{H}_{8}$ para determinar las propiedades de los gases de combustión a la entrada de la turbina de gas.

\begin{tabular}{|l|r|r|}
\hline \multicolumn{1}{|c|}{$\begin{array}{c}\text { Gas } \\
\text { Natural }\end{array}$} & Gases de combustión & \multicolumn{1}{c|}{ Aire } \\
\hline $\mathrm{cp},[\mathrm{kJ} / \mathrm{kgK}]$ & 1.3255 & 1.005 \\
\hline $\mathrm{R},[\mathrm{kJ} / \mathrm{kgK}]$ & 0.2914 & 0.28667 \\
\hline$\gamma,[-]$ & 1.2818 & 1.4 \\
\hline PCI, $[\mathrm{kJ} / \mathrm{kg}]$ & 49116.18 & - \\
\hline
\end{tabular}

Tabla 2 Propiedades de gases de combustión y aire

\section{Caldera de Recuperación de Calor}

Para el caso de cada configuración de ciclo combinado, se debe de considerar la diferencia de temperaturas de pinch point, $\Delta \mathrm{PP}$. Por lo tanto, en la Tabla 3 se muestran los valores de $\Delta$ PP para cada configuración de ciclo combinado.

\section{NP 2NP 3NP}

\begin{tabular}{|c|c|c|c|}
\hline$\Delta \mathrm{T}_{\mathrm{PPAP}},\left[{ }^{\circ} \mathrm{C}\right]$ & 30 & 30 & 90 \\
\hline$\Delta \mathrm{T}_{\mathrm{PPMP}},\left[{ }^{\circ} \mathrm{C}\right]$ & - & - & 40 \\
\hline$\Delta \mathrm{T}_{\mathrm{PPBP}},\left[{ }^{\circ} \mathrm{C}\right]$ & - & 50 & 40 \\
\hline
\end{tabular}

Tabla 3 Condiciones de operación de la CRC

Ciclo de vapor

Así mismo, en la Tabla 4 se muestran las condiciones de operación para el ciclo de vapor, para las tres configuraciones de ciclo combinado. Se muestra que para el caso de la presión de vapor vivo, $\mathrm{P}_{\mathrm{v} 1}$ se tiene la restricción de que, la calidad del vapor en la última etapa de expansión sea mayor o igual a 0.88 . 
Para el caso de la temperatura de vapor vivo, ésta debe ser menor a $580{ }^{\circ} \mathrm{C}$, también se considera que la temperatura de vapor vivo sea menor a la temperatura $\mathrm{T}_{\mathrm{g} 4}$, a partir de la definición de la diferencia de temperaturas de aproximación caliente, es decir, $\mathrm{T}_{\mathrm{v} 1}=\mathrm{T}_{\mathrm{g} 4-\Delta \mathrm{T}_{\mathrm{AC}} \mathrm{y}}$ que esta $\Delta \mathrm{T}_{\mathrm{AC}}$ sea siempre mayor o igual a $50^{\circ} \mathrm{C}$, ya que es necesario dicha diferencia para que exista una transferencia de calor de los gases hacia la corriente de vapor en el intercambiador de calor denominado sobrecalentador alta presión.

\begin{tabular}{|l|r|}
\cline { 2 - 2 } \multicolumn{1}{l|}{} & Ciclo de vapor \\
\hline $\mathrm{P}_{\mathrm{v} 1},[\mathrm{bar}]$ & $\mathrm{X}_{\mathrm{vi}} \geq 0.88$ \\
\hline $\mathrm{T}_{\mathrm{v} 1},\left[{ }^{\circ} \mathrm{C}\right]$ & $\mathrm{T}_{\mathrm{g} 4}>\mathrm{Tv} 1<580$ \\
\hline $\mathrm{P}_{\text {cond }},[\mathrm{bar}]$ & 0.07 \\
\hline$\eta_{\mathrm{SIT}},[-]$ & 0.9 \\
\hline $\mathrm{P}_{\text {baja }},[\mathrm{bar}]$ & 5 \\
\hline $\mathrm{P}_{\mathrm{rec}} / \mathrm{P}_{\mathrm{v} 1}$ & 0.3 \\
\hline
\end{tabular}

Tabla 4 Condiciones de operación del ciclo de vapor

Por otro lado, se utiliza el parámetro que relaciona la presión de recalentamiento con respecto a la presión de vapor vivo, $\mathrm{P}_{\mathrm{rec}} / \mathrm{P}_{\mathrm{v} 1}$, para el caso del ciclo combinado con tres niveles de presión debido que en esta configuración existe un recalentamiento del vapor, Figura 3.

\section{Metodología}

Análisis energético de turbina de gas

En la Figura 4 se muestra el diagrama Temperatura-entropía del ciclo de turbina de gas, para los tres casos mostrados en la Tabla 1. Se muestra el proceso de compresión (g1-g2), proceso de suministro de calor a presión constante (g2-g3) y finalmente el proceso de expansión en la turbina de gas (g3-g4).

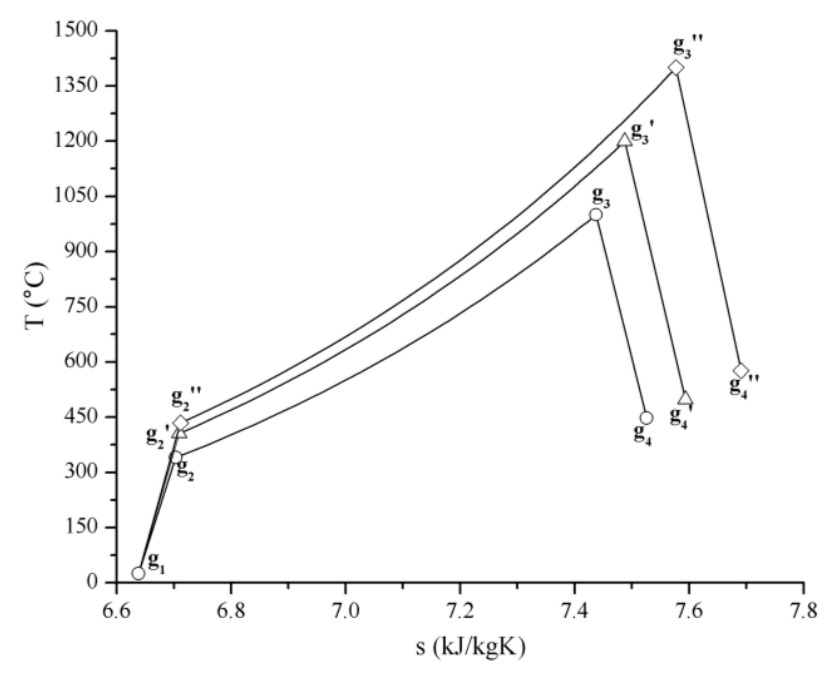

Figura 4 Diagrama T-s del ciclo de turbina de gas
La expresión para obtener el trabajo motor desarrollado en la turbina de gas en función de los parámetros descritos en las Tablas 1 y 2 es,

$$
W_{m}=c_{p_{a}} T_{g 1}\left[\begin{array}{l}
(1+r c a) \frac{c_{p_{g c}}}{c_{p_{a}}} y \eta_{S I T}\left(1-\frac{1}{\pi^{x g c}}\right) \\
\frac{1}{\eta_{S I C}}\left(\pi^{x a}-1\right)
\end{array}\right]
$$

Por otro lado, la expresión para obtener el calor suministrado en la cámara de combustión, para poder elevar desde la temperatura a la salida del compresor hasta la temperatura a la entrada de la turbina de gas, también en función de los parámetros descritos anteriormente es

$q_{\text {sum }}=c_{p_{s}} T_{g 1}\left[\begin{array}{c}(1+r c a) \frac{c_{p_{g c}}}{c_{p_{s}}} y \\ -1-\frac{1}{\eta_{S I C}}\left(\pi^{x a}-1\right)\end{array}\right]$ de gas es

$\eta_{T G}=\frac{W_{m}}{q_{\text {sum }}}$

El flujo de aire necesario para generar una potencia dada es

$$
\dot{m}_{a}=\frac{\dot{W}_{m T G}}{W_{m}}
$$

La cantidad de flujo de combustible requerido es

$$
\dot{m}_{c}=\frac{\dot{m}_{a} q_{\text {sum }}}{P C I}
$$

El Consumo Específico de Combustible, CEC, determina la cantidad de combustible necesario para generar un $\mathrm{kWh}$, 
$C E C=\frac{3600 \dot{m}_{c}}{\dot{W}_{m T G}}$

Los parámetros más importantes para el análisis en la CRC son el flujo de gases de combustión provenientes de la TG y la temperatura de los mismos, por lo tanto

$$
\begin{aligned}
& \dot{m}_{g c}=\dot{m}_{a}+\dot{m}_{c} \\
& T_{g 4}=T_{g 3}\left[1-\eta_{S I T}\left(1-\frac{1}{\pi^{x g c}}\right)\right]
\end{aligned}
$$

\section{Análisis en la Caldera de Recuperación de Calor}

La diferencia de temperaturas de pinch point se define para los domos de alta, media y baja presión de la siguiente manera

$$
\begin{aligned}
\Delta T_{P P_{A P}} & =T_{g 10}-T_{V 18} \\
\Delta T_{P P_{M P}} & =T_{g 13}-T_{V 23} \\
\Delta T_{P P_{B P}} & =T_{g 16}-T_{V 11}
\end{aligned}
$$

Así mismo la diferencia de temperaturas de aproximación caliente es

$$
\Delta T_{A C}=T_{g 4}-T_{V 1}
$$

Se hace un balance de energía en los diferentes intercambiadores de calor que integran a la CRC para determinar las temperaturas intermedias, así como el flujo de vapor generado en el ciclo de vapor. Para este caso, únicamente se muestra el balance de energía del ciclo combinado con tres niveles de presión, ya que existe una similitud con respecto a las configuraciones del ciclo combinado de uno y dos niveles de presión.

El balance de energía realizado a la sección de alta presión es

\section{SCAP2}

$\dot{m}_{g C}\left(h_{g 4}-h_{g 5}\right)=\dot{m}_{V_{A P}}\left(h_{V 1}-h_{V 2}\right)$

\section{SCAP1}

$\dot{m}_{g c}\left(h_{g 6}-h_{g 7}\right)=\dot{m}_{V_{A P}}\left(h_{V 20}-h_{V 19}\right)$

\section{EVAP}

$$
\dot{m}_{g c}\left(h_{g 8}-h_{g 9}\right)=\dot{m}_{V_{A P}}\left(h_{v 19}-h_{v 18}\right)
$$

ECAP2

$$
\dot{m}_{g c}\left(h_{g 9}-h_{g 10}\right)=\dot{m}_{V_{A P}}\left(h_{v 18}-h_{V 17}\right)
$$

El balance de energía realizado a la sección de presión media es

RCMP2

$$
\dot{m}_{g c}\left(h_{g 5}-h_{g 6}\right)=\left(\dot{m}_{V_{A P}}+\dot{m}_{V_{M P}}\right)\left(h_{V 5}-h_{V 4}\right)
$$

RCMP1

$$
\dot{m}_{g c}\left(h_{g 7}-h_{g 8}\right)=\left(\dot{m}_{V_{A P}}+\dot{m}_{V_{M P}}\right)\left(h_{V 4}-h_{v 3}\right)
$$

SCMP

$$
\dot{m}_{g c}\left(h_{g 10}-h_{g 11}\right)=\dot{m}_{V_{M P}}\left(h_{v 25}-h_{v 24}\right)
$$

EVMP

$$
\dot{m}_{g c}\left(h_{g 12}-h_{g 13}\right)=\dot{m}_{V_{M P}}\left(h_{v 24}-h_{v 23}\right)
$$

En la Figura 3 se muestran dos intercambiadores de calor ubicados en una misma posición el ECAP1 y ECMP, por lo tanto, realizando un balance de energía se tiene

$$
\dot{m}_{g c}\left(h_{g 14}-h_{g 15}\right)=\dot{m}_{v_{A P}}\left[\begin{array}{l}
\left(h_{v 17}-h_{v 16}\right)+ \\
\left(\frac{\dot{m}_{v_{M P}}}{\dot{m}_{V_{A P}}}\right)\left(h_{v 23}-h_{v 22}\right)
\end{array}\right]
$$
presión

Finalmente para la sección de baja

SCBP2

$\dot{m}_{g c}\left(h_{g 11}-h_{g 12}\right)=\dot{m}_{V_{B P}}\left(h_{v 14}-h_{v 13}\right)$

SCBP1

$\dot{m}_{g c}\left(h_{g 13}-h_{g 14}\right)=\dot{m}_{V_{B P}}\left(h_{v 13}-h_{v 12}\right)$

EVBP

$$
\dot{m}_{g c}\left(h_{g 15}-h_{g 16}\right)=\dot{m}_{V_{B P}}\left(h_{v 12}-h_{v 11}\right)
$$

ECBP

$$
\dot{m}_{g c}\left(h_{g 16}-h_{g 17}\right)=\left(\dot{m}_{v T}\right)\left(h_{v 11}-h_{v 10}\right)
$$

Donde el $\dot{m}_{V T}=\dot{m}_{V_{A P}}+\dot{m}_{V_{M P}}+\dot{m}_{V_{B P}}$ 
Finalmente, para el caso del ciclo combinado con tres niveles de presión, se presentan un número de 13 ecuaciones con 13 incógnitas las cuales se pueden resolver a partir de algún método numérico o alguna herramienta computacional.

Por consiguiente, para el caso del ciclo combinado con uno y dos niveles de presión, se debe emplear esta misma metodología para determinar tanto las temperaturas intermedias en la CRC y los flujos de vapor.

\section{Análisis del ciclo de vapor}

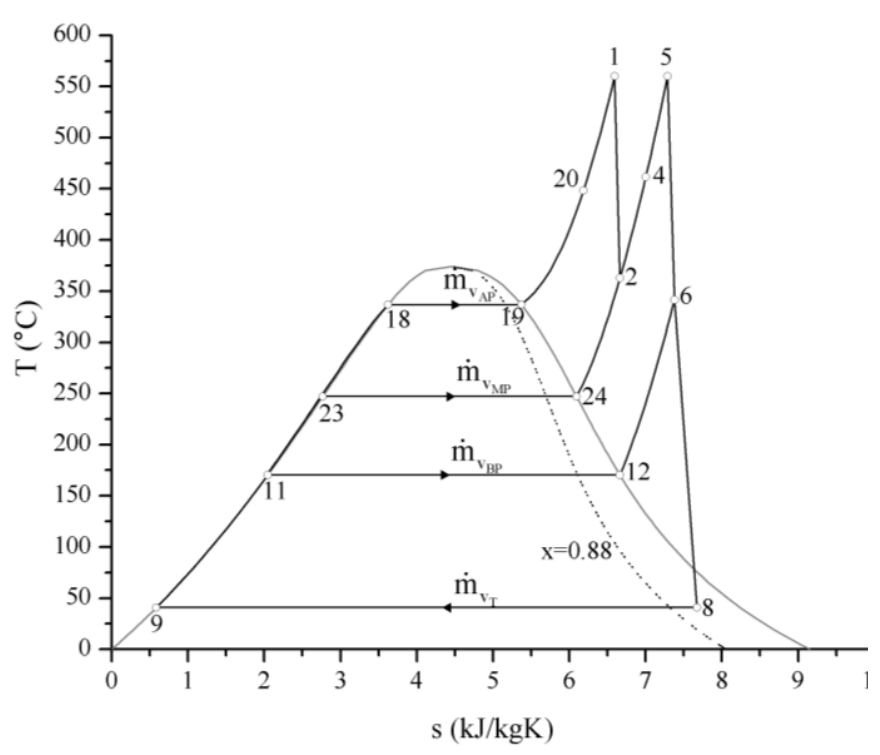

Figura 5 Diagrama T-s del ciclo de vapor CC tres niveles

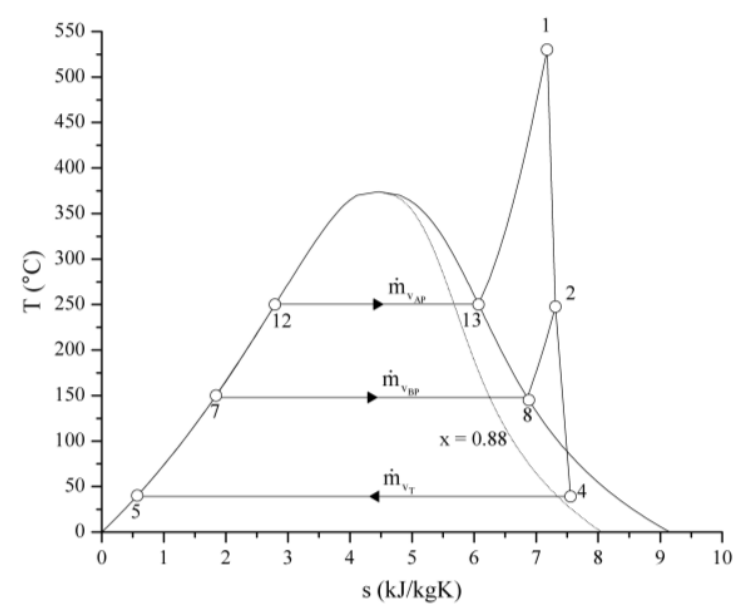

Figura 6 Diagrama T-s del ciclo de vapor CC tres niveles

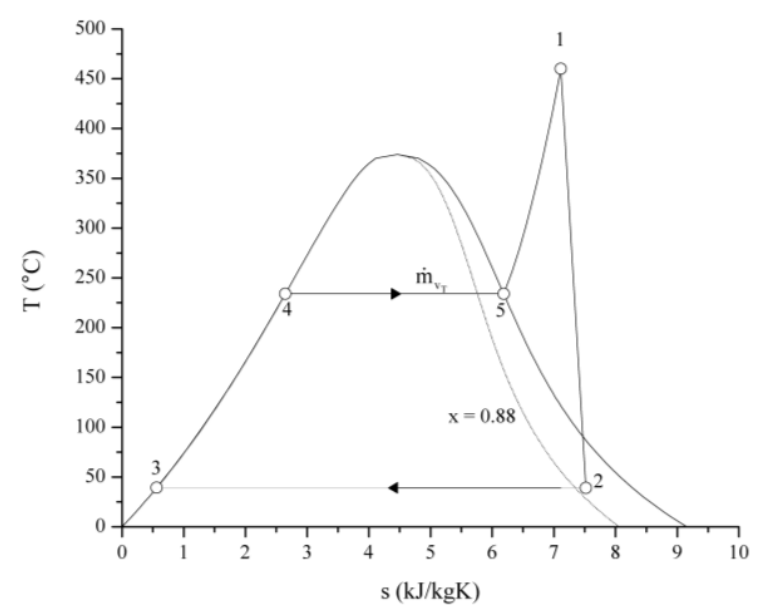

Figura 7 Diagrama T-s del ciclo de vapor CC un nivel

En las Figuras 5 a 7 se muestran los diagramas temperatura-entropía de los ciclos de vapor para los ciclos combinados de uno, dos y tres niveles de presión. En la Figura 7 se muestra el ciclo de vapor acoplado a la CRC con un nivel de presión, muestra que solamente tiene una etapa de expansión, mientras que, para el caso de la configuración con dos niveles, Figura 6 , se tienen dos etapas de expansión y finalmente para la configuración de tres niveles se tiene un mayor número de etapas de expansión (Figura $5)$.

A partir de las configuraciones de los ciclos combinados con uno, dos y tres niveles de presión mostrada en las Figuras 1 a 3 , se determinan los siguientes parámetros de desempeño.

Realizando un balance de energía en las secciones de la turbina de vapor y en las bombas, se puede determinar la potencia generada por el ciclo, por lo tanto

$$
\dot{W}_{m_{T V C C}}=\sum \dot{W}_{T V}-\sum \dot{W}_{B}
$$

Para el caso del ciclo combinado con un nivel de presión. La potencia generada en la turbina de vapor es

$$
\dot{W}_{T V_{C C 1}}=\dot{m}_{V_{T}}\left(h_{V 1}-h_{V 2}\right)
$$

La potencia suministrada a la bomba es

$$
\dot{W}_{B_{C C 1}}=\dot{m}_{V_{T}}\left(h_{V 4}-h_{V 3}\right)
$$

Para determinar la potencia motor en el ciclo combinado con dos niveles de presión, se 
debe calcular la potencia generada en las secciones de la turbina de vapor.

$\dot{W}_{T V_{C C 2}}=\dot{m}_{V_{A P}}\left[\begin{array}{l}\left(h_{V 1}-h_{V 2}\right)+ \\ \left(1+\frac{\dot{m}_{V_{B P}}}{\dot{m}_{V_{A P}}}\right)\left(h_{V 3}-h_{V 4}\right)\end{array}\right]$

Y la potencia de las bombas es

$$
\dot{W}_{B_{C C 2}}=\dot{m}_{V_{A P}}\left[\begin{array}{l}
\left(h_{v 11}-h_{V 10}\right)+ \\
\left(1+\frac{\dot{m}_{V_{B P}}}{\dot{m}_{V_{A P}}}\right)\left(h_{V 6}-h_{V 5}\right)
\end{array}\right]
$$

Finalmente, la potencia generada en las diferentes secciones de la turbina de vapor y la potencia suministrada a las diferentes bombas para el ciclo combinado de tres niveles de presión son:

La potencia total generada en la turbina es:

$$
\dot{W}_{T_{C C 3}}=\dot{m}_{V_{A P}}\left[\begin{array}{l}
\left(h_{V 1}-h_{V 2}\right)+ \\
\left(1+\frac{\dot{m}_{V_{B P}}}{\dot{m}_{V_{A P}}}\right)\left(h_{V 6}-h_{V 5}\right)+ \\
\left(1+\frac{\dot{m}_{V_{M P}}}{\dot{m}_{V_{A P}}}+\frac{\dot{m}_{V_{B P}}}{\dot{m}_{V_{A P}}}\right)\left(h_{V 8}-h_{V 7}\right)
\end{array}\right]
$$

Y la potencia total en las bombas es

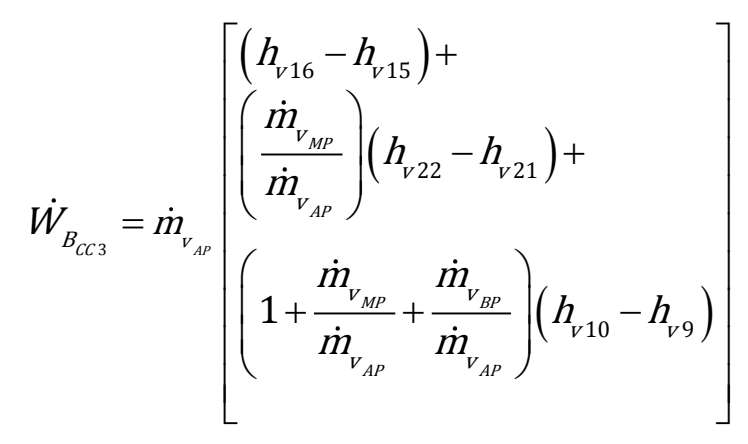

A continuación, se presentan otros parámetros de desempeño, los cuales se muestran de manera generalizada para las tres configuraciones de ciclo combinado.

El flujo de calor cedido por los gases de combustión hacia el ciclo de vapor se determina también a partir de un balance de energía en la CRC, la cual de manera generalizada es

$$
\dot{Q}_{s u m_{C C i}}=\dot{m}_{g c_{i}} c_{p_{g c_{i}}} \sum_{j=1}^{n}\left(T_{g_{j}}-T_{g_{j+1}}\right)
$$

Donde i representa la configuración de ciclo combinado de uno, dos o tres niveles de presión, j representa la sumatoria de las diferencias de temperaturas de los intercambiadores de calor en la CRC.

La eficiencia térmica del ciclo de vapor para cualquiera de las tres configuraciones analizadas relaciona la potencia generada con respecto al flujo de calor cedido por los gases en la CRC, por lo tanto,

$$
\eta_{T V_{C C i}}=\frac{\dot{W}_{m_{T V C i}}}{\dot{Q}_{\text {Sum }}}
$$

El Consumo Específico de Vapor, CEV, determina la cantidad de vapor necesaria para poder generar un $\mathrm{kWh}$,

$$
C E V_{C C i}=\frac{3600 \dot{m}_{V_{C C i}}}{\dot{W}_{m_{T V C C i}}}
$$

\section{Análisis del ciclo combinado}

Finalmente, la potencia total generada por el ciclo combinado es la sumatoria de las potencias de ambos ciclos, por lo tanto y de manera generalizada para el ciclo combinado es

$$
\dot{W}_{C C i}=\dot{W}_{m T G}+\dot{W}_{m T V_{C C i}}
$$

La eficiencia térmica de ciclo combinado, en función de las eficiencias térmicas de cada ciclo se expresa

$$
\eta_{C C i}=\eta_{T G i}+\eta_{T V_{C C i}}-\left(\eta_{T G i} \eta_{T V_{C C i}}\right)
$$

El Consumo Térmico Unitario se define como la cantidad de energía necesaria para generar un $\mathrm{kWh}$, entonces

$$
C T U=\frac{3600}{\eta_{C C i}}
$$

Finalmente el Consumo Específico de Combustible para el ciclo combinado es 


$$
C E C_{C C i}=\frac{3600 \dot{m}_{c}}{\dot{W}_{C C i}}
$$

\section{Resultados y Discusión}

A partir de las condiciones de operación de tres diferentes tipos de turbinas de gas mostradas en la Tabla 1, en la Figura 8 se muestra una comparación de la eficiencia térmica obtenida, en donde el mayor valor se obtiene para la TG3, con una eficiencia de 0.33 . Dicho incremento se traduce en un mayor aprovechamiento de la energía térmica del combustible, esto como consecuencia de un incremento en la relación de presiones y la temperatura de los gases a la entrada de la turbina de gas.

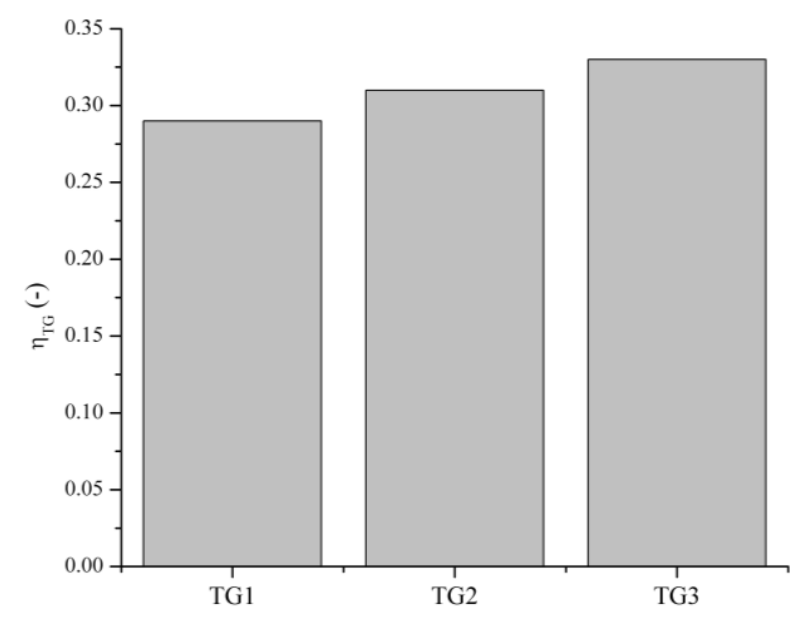

Figura 8 Eficiencia térmica para los tres casos de TG

En la Figura 9 se muestra un aumento de $100^{\circ} \mathrm{C}$ en la temperatura de los gases salientes de la turbina de gas al pasar de TG1 a TG3. Con respecto al trabajo motor generado, pasa de 329 $\mathrm{kJ} / \mathrm{kg}$ con la TG1, a $500 \mathrm{~kJ} / \mathrm{kg}$ con la TG3. Como consecuencia, se tendrá una disminución en el flujo de los gases de combustión para generar la misma potencia.

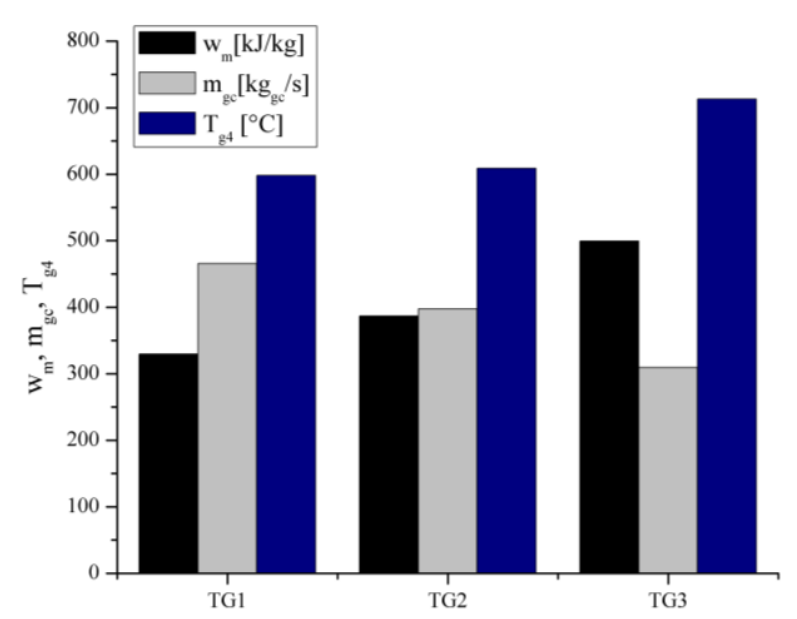

Figura 9 Parámetros de desempeño para los tres casos TG

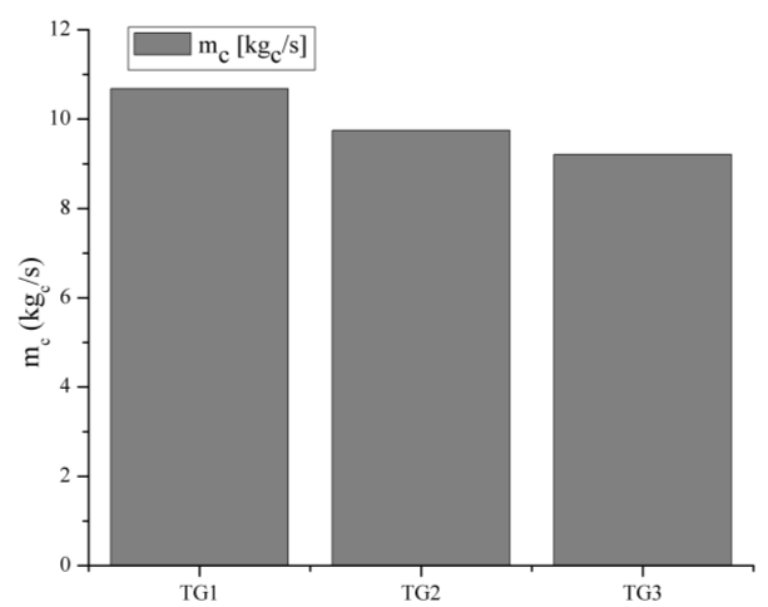

Figura 10 Flujo de combustible para los tres casos de TG

Así mismo en el consumo de combustible utilizado, en la Figura 10 se muestra que existe una disminución de $1.5 \mathrm{~kg} / \mathrm{s}$ al pasar de TG1 a TG3, lo cual resulta ser benéfico en el sentido de un ahorro de combustible y un menor impacto ambiental. Finalmente, esta disminución del combustible suministrado para satisfacer una demanda de potencia también puede verse como una menor cantidad de energía necesaria para poder producir un $\mathrm{kWh}$, lo que trae consigo una disminución en el costo de generación de un $\mathrm{kW}$ eléctrico.

En la Figura 11 se puede mostrar que, optar por un tipo de turbina de gas con mejores características tecnológicas, en este caso trae consigo una disminución del CEC. Si bien la energía que contienen los gases de combustión salientes de la turbina de gas no puede ser nuevamente usados en esta máquina térmica, obtener el máximo aprovechamiento de este recurso para la producción de una potencia adicional en el ciclo de vapor resulta ser lo primordial.

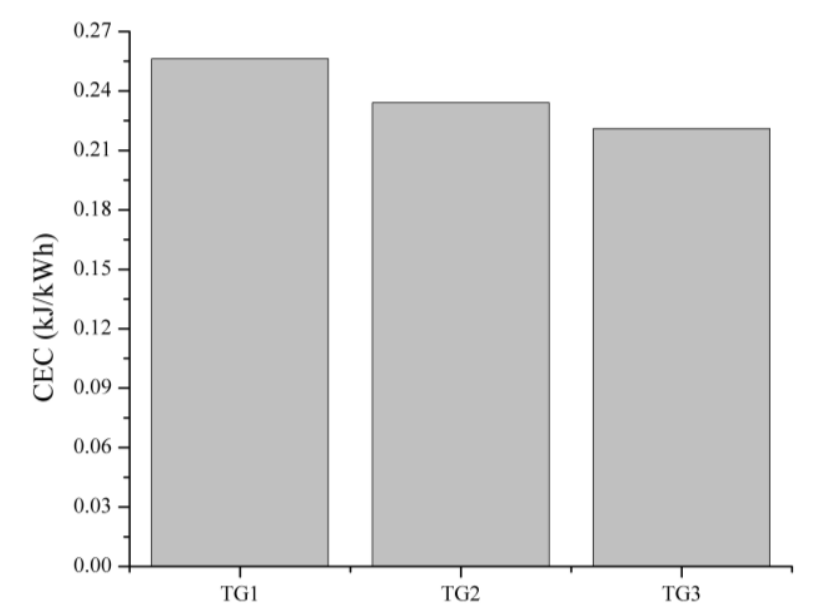

Figura 11 Consumo Específico de Combustible para los tres casos de TG

MÉNDEZ-CRUZ, Ladislao Eduardo, SALAZAR-PEREYRA, Martín, LUGO-LEYTE, Raúl y PÉREZ-BEDOLLA, Rafael. Análisis termodinámico de las condiciones de operación de los ciclos combinados turbina de gas-vapor con uno, dos y tres niveles de presión. Revista de Ingeniería Mecánica. 2019. 
Por lo tanto, el enfoque que se debe dar al ciclo combinado es, para obtener la máxima producción de potencia y no necesariamente para la máxima eficiencia. Cabe señalar que se debe tomar en cuenta la restricción de la calidad del vapor en la última etapa de expansión en la turbina de vapor, la cual no debe de ser mayor a 0.88. Por lo tanto, en la Figura 12 se muestra la potencia de ciclo combinado de un nivel de presión en función de la calidad del vapor, en donde básicamente se muestra que, el área de operación segura del ciclo de vapor para cualquier temperatura de vapor vivo, se tiene para una presión menor a 40 bar.

También se muestra que a media que se va incrementando la condición de presión, existe una reducción en el rango de temperaturas de vapor vivo, por lo tanto, para este caso, la máxima potencia que se puede obtener es de aproximadamente $240 \mathrm{MW}$ necesariamente con una temperatura que tenga como valor mínimo $500^{\circ} \mathrm{C}$ y una presión que no sea superior a 60 bar.

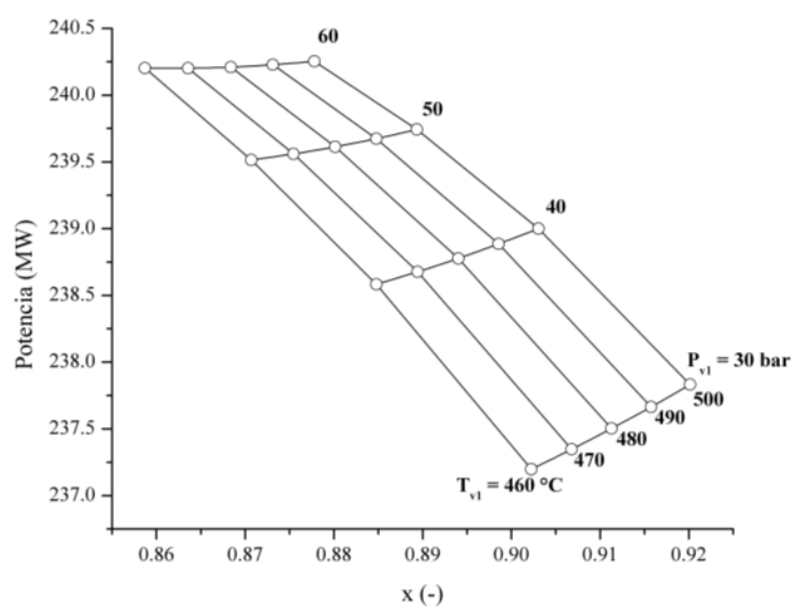

Figura 12 Potencia de $\mathrm{CC}$ un nivel en función de la calidad de vapor

Para la configuración mostrada en la Figura 2 de un ciclo combinado con dos niveles de presión, a partir del análisis mostrado en la Figura 13 se muestra que las condiciones de operación de la turbina de vapor se pueden incrementar hasta 60 bar y $530^{\circ} \mathrm{C}$.

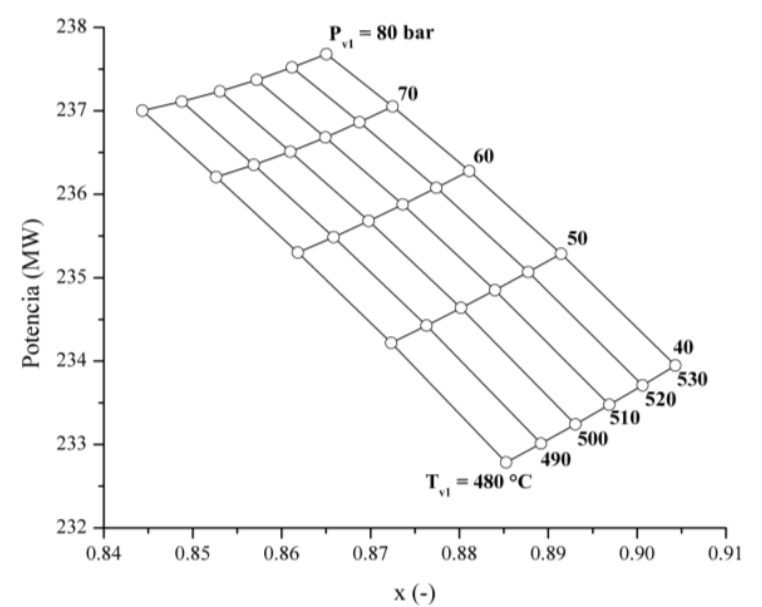

Figura 13 Potencia de $\mathrm{CC}$ dos niveles en función de la calidad de vapor

Sin embargo, la infraestructura de los ciclos combinados para la generación de potencia en México es con base a la configuración de tres niveles de presión, las cuales se ha optado por la implementación de turbinas de gas de última tecnología, similares a las del caso TG3.

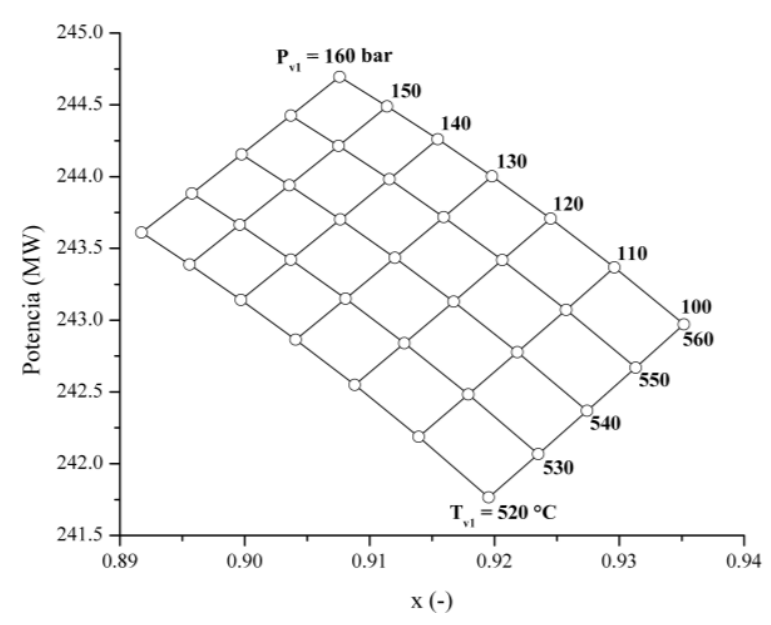

Figura 14 Potencia de CC tres niveles en función de la calidad de vapor

Por lo tanto, en la Figura 14 se muestra que al implementar un tercer domo, se tiene un mayor rango de operación seguro, así mismo de incrementar la potencia hasta $244.75 \mathrm{MW}$ para una presión de 160 bar y una temperatura de $560^{\circ} \mathrm{C}$.

La razón por la cual se tenga una mayor área de operación segura del ciclo de vapor en comparación con las otras dos configuraciones de ciclo combinado se debe a que se tiene un recalentamiento en la sección de media presión. Finalmente en las Figuras 15 a 17 se muestra la eficiencia de ciclo combinado en función de la calidad del vapor, para las configuraciones de uno, dos y tres niveles de presión. 


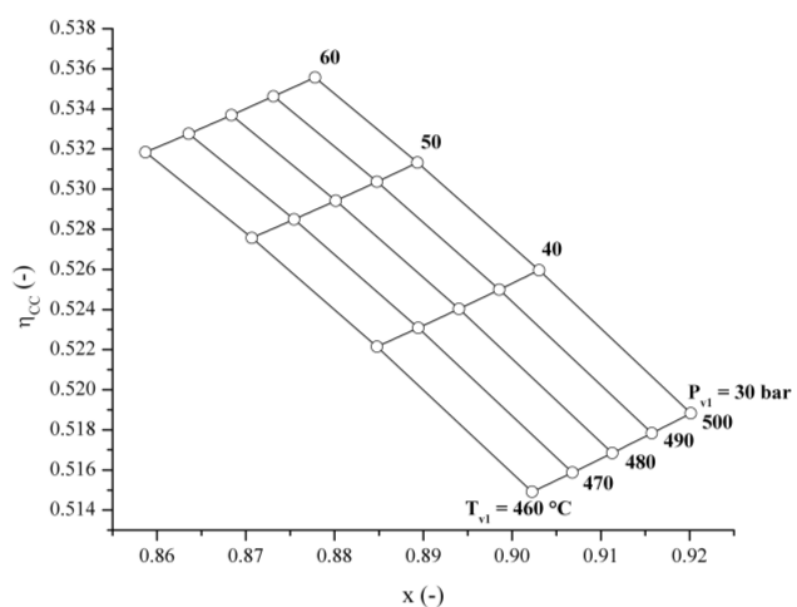

Figura 15 Eficiencia de $\mathrm{CC}$ en función de la calidad de vapor $\mathrm{CC}$ un nivel

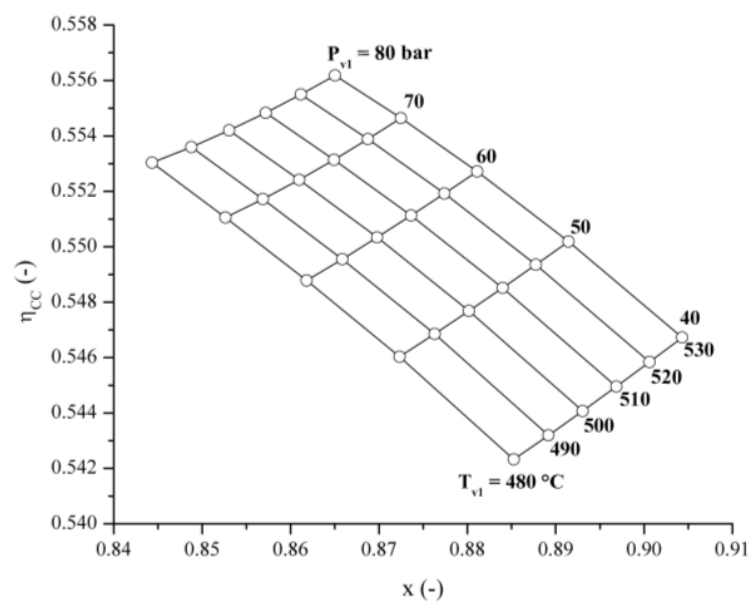

Figura 16 Eficiencia de $\mathrm{CC}$ en función de la calidad de vapor CC dos niveles

La eficiencia del ciclo combinado se incrementa a medida que se tiene un mayor nivel de presion, considerando las mismas condiciones de operación de presion y temperatura de vapor vivo descritas en las Figuras 15 a 17. Por consiguiente, la máxima eficiencia que se tiene con el ciclo combinado de un nivel de presion es de aproximadamente 0.53 , mientras que para el caso de dos niveles de presion se incrementa la eficiencia en 2 puntos porcentuales.

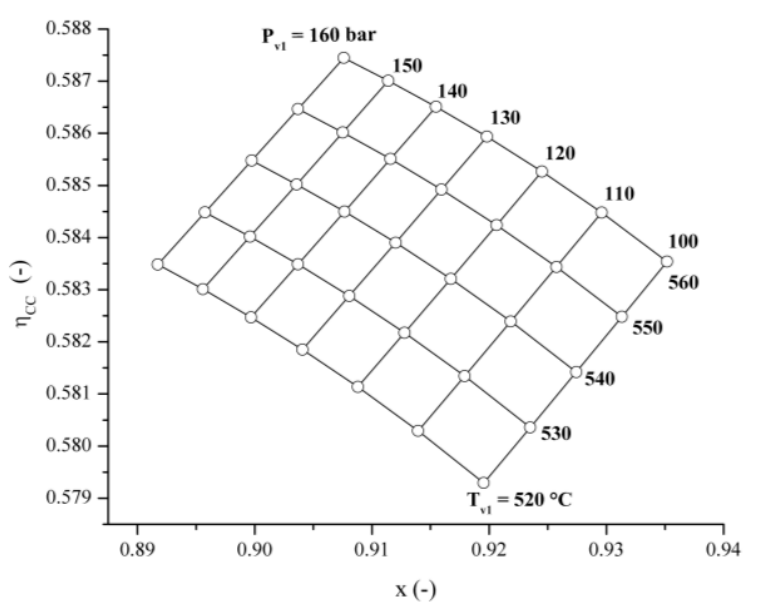

Figura 17 Eficiencia de $\mathrm{CC}$ en función de la calidad de vapor CC tres niveles
Finalmente para la configuracion de ciclo combinado con tres niveles de presion se logran alcanzar eficiencias entre 0.58 y 0.59 , eficiencias térmicas altas en comparación con cualquier otro tipo de central termica para la generación de potencia. En la Figura 18 se muestra un comparativo de la eficiencia térmica obtenida a partir del análisis de turbina de gas y ciclo de vapor de manera independiente y finalmente la eficiencia de ciclo combinado. Se muestra que, las eficiencias térmicas de la TG y del ciclo de vapor en promedio están en 0.33 , mientras que si funcionan como ciclo combinado, se pueden lograr alcanzar eficiencias muy cercanas a 0.60 .

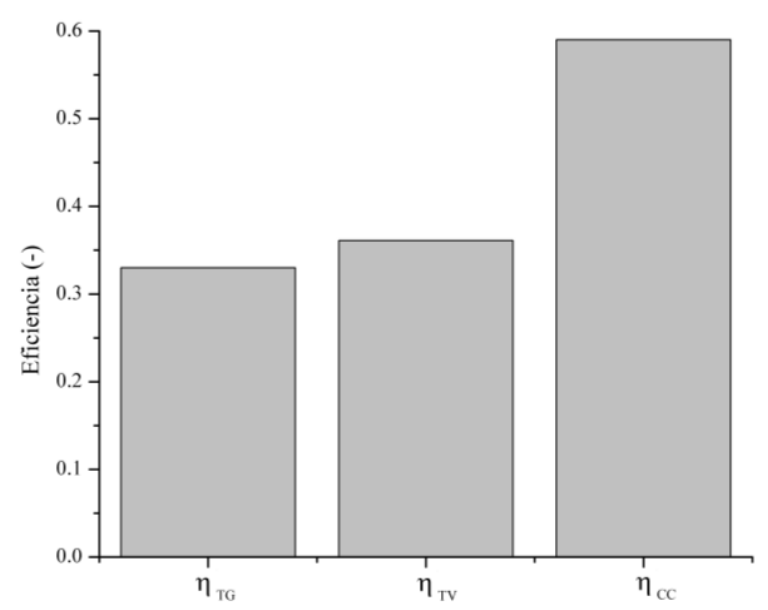

Figura 18 Comparación de la eficiencia para CC tres niveles de presión

Otra forma de observar el beneficio que trae consigo implementar ciclos combinados con tres niveles de presión es a partir del análisis del CTU. En la Figura 19 se muestra dicho parámetro nuevamente para los ciclos desarrollados de manera independiente y ambos operando bajo un ciclo combinado, se muestra que para el caso de TG y TV la cantidad de energía necesaria para generar un $\mathrm{kWh}$ sobrepasa las 10,000 unidades, mientras que para el caso del ciclo combinado existe una disminución de la cantidad de energía hasta de un $50 \%$. 


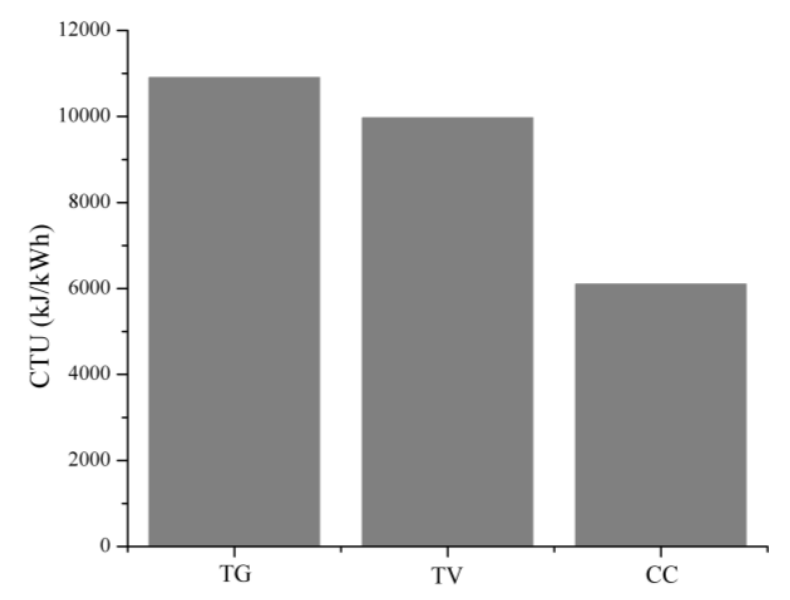

Figura 19 Comparación del Consumo Térmico Unitario en para CC tres niveles de presión

\section{Conclusiones}

Los sistemas de generación de energía con ciclos combinados turbina de gas-vapor en comparación con el ciclo de turbina de gas y ciclo de vapor de manera independiente, son los que presentan el mejor comportamiento de acuerdo al análisis termodinámico realizado. Así mismo la configuración de ciclo combinado que tuvo las mejores condiciones de potencia y eficiencia térmica fue con respecto a 3 niveles de presión.

Por lo tanto, para México resulta una opción viable y certera, la implementación de tecnologías de ciclo combinado turbina de gasvapor con tres niveles de presión para poder cubrir con la demanda actual de generación de potencia requerida así como tener la seguridad de que el incremento anual en la demanda de energía no sea un problema.

\section{Referencias}

Ataei, A., \& Yoo, C. (2010). Combined pinch and exergy analysis for energy efficiency optimization in a steam power plant. International Journal of Physical Sciences, 5(7), 1110-1123.

Cihan, A., Hacıhafizog `lu, O., \& Kahveci, K. (2006). Energy-exergy analysis and modernization suggestions for a combinedcycle power plant. International Journal of Energy Research, 30(2), 115-126.

Franco, A., \& Russo, A. (2002). Combined cycle plant efficiency increase based on the optimization of the heat recovery steam generator operating parameters. International Journal of Thermal Sciences, 41(9), 843-859.
Ganjehkaviri, A., Jaafar, M. M., \& Hosseini, S. E. (2015). Optimization and the effect of steam turbine outlet quality on the output power of a combined cycle power plant. Energy conversion and management, 89, 231-243.

Kaviri, A. G., Jaafar, M. N. M., Lazim, T. M., \& Barzegaravval, H. (2013). Exergoenvironmental optimization of heat recovery steam generators in combined cycle power plant through energy and exergy analysis. Energy conversion and management, 67, 27-33.

Lugo-Leyte, R., Salazar-Pereyra, M., BonillaBlancas, A. E., Lugo-Méndez, H. D., RuízRamírez, O. A., \& Toledo-Velázquez, M. (2016). Exergetic Analysis of Triple-Level Pressure Combined Power Plant with Supplementary Firing. Journal of Energy Engineering, 142(4), 05016001.

Mansouri, M. T., Ahmadi, P., Kaviri, A. G., \& Jaafar, M. N. M. (2012). Exergetic and economic evaluation of the effect of HRSG configurations on the performance of combined cycle power plants. Energy Conversion and Management, 58, 47-58.

Secretaría de Energía. (2018). Balance Nacional de Energía.

Secretaría de Energía. (2018). Prospectiva del Sector Eléctrico 2018-2032.

Velazquez, M. T., Topete, M. P., Flores, M. A. M., Silva, F. S., Leyte, R. L., \& Fancis, J. A. (2016). Análisis del comportamiento térmico en la planta termoeléctrica Valle de México mediante pruebas de evaluación en turbinas de gas. DYNA Energía y Sostenibilidad, 5(1).

Zhang, G., Zheng, J., Yang, Y., \& Liu, W. (2016). Thermodynamic performance simulation and concise formulas for triplepressure reheat HRSG of gas-steam combined cycle under off-design condition. Energy conversion and management, 122, 372-385. 